\title{
Modified Overall Immune Related Response Criterion, Complete Remission
}

National Cancer Institute

\section{Source}

National Cancer Institute. Modified Overall Immune Related Response Criterion,

Complete Remission. NCI Thesaurus. Code C111084.

The evaluation of the antitumor response shows a complete disappearance of all lesions (whether measurable or not) and no new lesions. All measurable lymph nodes also have a reduction in short axis diameter to $10 \mathrm{~mm}$. 